EPJ Web of Conferences 108, 02023 (2016)

DOI: $10.1051 /$ epjconf/201610802023

(C) Owned by the authors, published by EDP Sciences, 2016

\title{
A New Segment Building Algorithm for the Cathode Strip Chambers in the CMS Experiment
}

\author{
I. Golutvin ${ }^{1}$, V. Karjavin ${ }^{1}$, V. Palichik ${ }^{1}$, N. Voytishin ${ }^{1, a}$, and A. Zarubin ${ }^{1}$ \\ ${ }^{1}$ Joint Institute for Nuclear Research, \\ Joliot-Curie 6, 141980 Dubna, Moscow Region, Russia
}

\begin{abstract}
A new segment building algorithm for the Cathode Strip Chambers in the CMS experiment is presented. A detailed description of the new algorithm is given along with a comparison with the algorithm used in the CMS software. The new segment builder was tested with different Monte-Carlo data samples. The new algorithm is meant to be robust and effective for hard muons and the higher luminosity that is expected in the future at the LHC.
\end{abstract}

\section{Introduction}

The CMS muon system should provide a good efficiency of the muon track reconstruction with a high spatial resolution. It is necessary to achieve the required momentum resolution [1]. The muon detector is located behind the hadronic calorimeter. The high energy muons themselves produce a significant number of electromagnetic (e.m.) secondaries passing through the calorimeter and the iron yoke matter. A part of the e.m. secondaries (especially from hard muons) reaches the muon detectors and makes the muon track reconstruction more difficult.

The aim of this paper is to present a new Cathode Strip Chamber (CSC) segment building algorithm and to analyze the results obtained by using it for different Monte-Carlo datasets.

The new segment building algorithm is meant to increase the efficiency and the accuracy of the segment reconstruction and improve the trajectory of the muons in the endcap muon system. It must be robust and stable at big luminosities and for high momentum values of muons up to $\mathrm{TeV}$ region.

\section{Segment builder in the Cathode Strip Chambers}

The endcap muon system consists of several stations. Each station consists of many CSCs. The CSC is the main type of detectors used in the CMS endcap muon system. Each CSC consists of six layers. The scheme of one of the layers is presented in the Figure 1.

The $z$-axis on all the pictures represents the axis oriented along the beam line. This means that each layer has its own distinct $z$-coordinate. Each layer consists of arrays of positively charged "anode" wires in a gas volume between negatively charged copper "cathode" strips. When muons pass through the gas volume, they knock electrons off the gas atoms creating an avalanche of electrons, which flock

\footnotetext{
a e-mail: nikolay.voytishin@cern.ch
} 


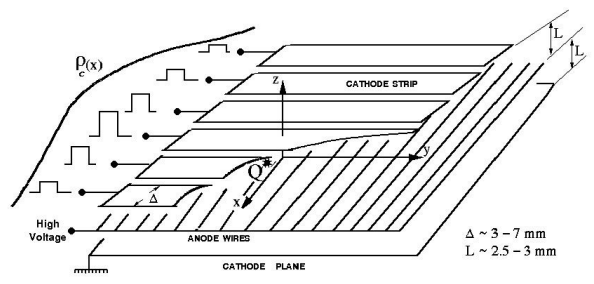

Figure 1: The principle of working of a Cathode Strip Chamber

to the anode wires. Positive ions move away from the wire and towards the copper cathode, inducing a charge pulse in the strips. Because the wires and the strips are perpendicular, two position coordinates are recorded for each passing particle and it is considered that a reconstructed hit (shortly "rechit") was registered on this layer. The radial coordinate (a rough measurement) is reconstructed from the anode information. The azimuthal coordinate (a precise measurement) is reconstructed from the cathode information with a spatial resolution of 100-200 microns. Having 6 layers with rechits registered on some of them it is possible to reconstruct a track-segment in a particular chamber.

The "standard" CSC segment building algorithm which is used in the current CMS software releases is based on the spanning tree approach. Each reasonable combination of hits is evaluated. The best possible segment candidates are chosen using variables sensitive to angular offsets between lines connecting hits from neighboring chamber layers in both projections. This is achieved by minimizing the sum of absolute values of the differences in angle slopes between neighboring layers.

As it will be described below, this algorithm gives an insufficient efficiency in the reconstruction of the high momentum muons which generate a big amount of background hits. As a result we get many short size segments.

\section{New CSC segment builder algorithm}

The proposed algorithm is built in a way that both coordinates (rough and precise) are taken into account. The Base Road approach [2] is the basic idea of the new algorithm. A schematic representation of the algorithm is shown in the Figure 2. The two most distant in $z$-coordinate layers with hits are chosen as the base layers. A pair of hits from base layers is chosen as a base pair for the future segment in such a way that the building segment points towards the interaction point. In Figure 2 the region for the base pair is shown in dotted line. This region is singled out of the experimental setup geometry [3] for each pair of base layers. In such a way we get rid of most of the fake short segments that can be built out of the background hits. The pair of chosen hits will be the base pair for the future segment. An imaginary line is traced through these base hits. CSCs are located in a strong magnetic field, however the approximation of the particle trajectory by a straight line in a single chember is admissible because of a very small thickness of the chamber in comparison to the whole trajectory length. New hits from the inner layers that are in the formed "base road" (dashed line) are added to the segment. If there is more than one hit that satisfies the cut on the coordinates imposed by the road more than one segment is built.

After all possible segments for the two base layers are built, the segments are assembled in groups. Each group is formed out of the segments that have common hits. Out of each group only the segment with the lowest $\chi^{2}$ [4] is chosen. If the chosen segment doesn't pass the $\chi^{2}$ threshold, the optimal rejection algorithm is used. This algorithm is meant to get rid of the hit that is the farthest from a straight line fit (Figure 3 ) by minimizing the sum of the absolute values of the deviations, 


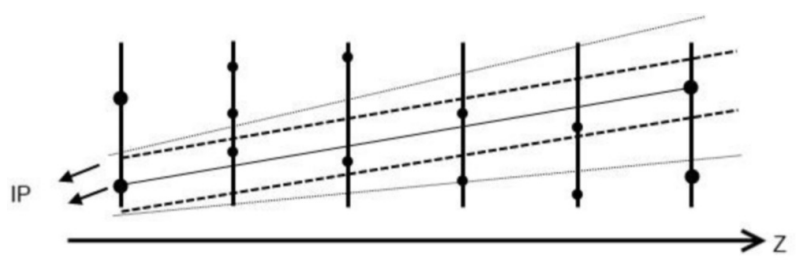

Figure 2: Modified Base Road approach

$$
\min \left(\sum_{i}\left|d_{i}\right|\right) .
$$

Once the segment is built, its hits are marked as used in order not to be considered in future iterations.

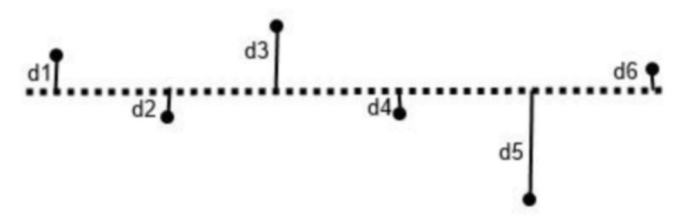

Figure 3: Optimal point rejection

\section{Results and analysis}

The new segment builder was checked on different official and private Monte-Carlo data samples with the aim to prove that it reconstructs the segments properly for all types of physical objects that might be produced from the beam collisions at the LHC. In this paper the results obtained with the new algorithm are compared with the results yielded by the standard segment builder.

The following Monte-Carlo dataset types were used for comparison:

- Official simulation samples:

- $Z$ boson decay in two muons $(Z \rightarrow \mu \mu)$;

$-Z^{\prime}$ boson with a mass of $2250 \mathrm{GeV} / \mathrm{c}^{2}$ decay in two muons $\left(Z^{\prime} \rightarrow \mu \mu\right)$.

- Private Monte-Carlo endcap single muon datasets with different values of transverse momentum $P_{t}=\sqrt{p_{x}^{2}+p_{y}^{2}}$

$-P_{t}=100 \mathrm{GeV} / \mathrm{c}$

$-P_{t}=1000 \mathrm{GeV} / \mathrm{c}$.

Both algorithms show good results for events with a small amount of e.m. secondaries and low momentum of muons. This type of events will not be considered in this paper. The results differ under the increase of the muon momentum and luminosity.

It is crucial to have a reasonable number of reconstructed segments in an event. This influences the speed and precision of the next trajectory reconstruction steps that come after the segment building procedure. The total number of segments reconstructed in a particular station per event is shown 
in the Figure 4. Black color on all the histograms shows the values for the new algorithm and the gray stands for the standard algorithm. The standard algorithm gives a high multiplicity of the reconstructed segments especially for high $P_{t}$ muons sometimes even exceeding the histogram maximum (60 segments). The new algorithm holds the maximum value of the segments in station per event around 20. There is also another observation that can be made out of these histograms. The first bin shows the amount of stations where the simulated muon passed through but no segments were reconstructed. The number of such cases is smaller for the new algorithm for all datasets.
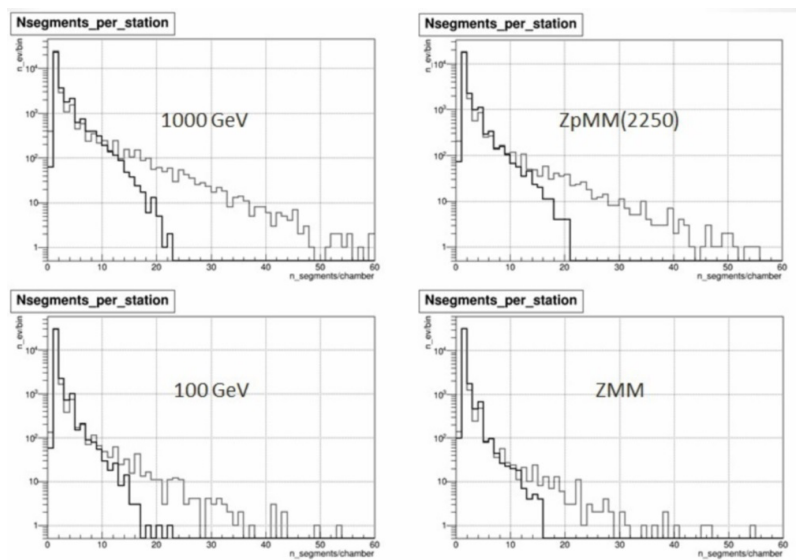

Figure 4: Segment multiplicity in a CSC station per event for simulated data

The number of hits per segment is presented in the Figure 5. Here all the reconstructed segments are taken into the account. We managed to decrease the number of 3-hit segments while the number of 6-hit segments increased. The fraction of the 6-hit segments identified by the new algorithm is about $60 \%$ for all the MC samples, while decreasing for the standard algorithm from $42 \%$ for $Z \rightarrow \mu \mu$ sample, down to $24 \%$ for $\mathrm{TeV}$ muons.
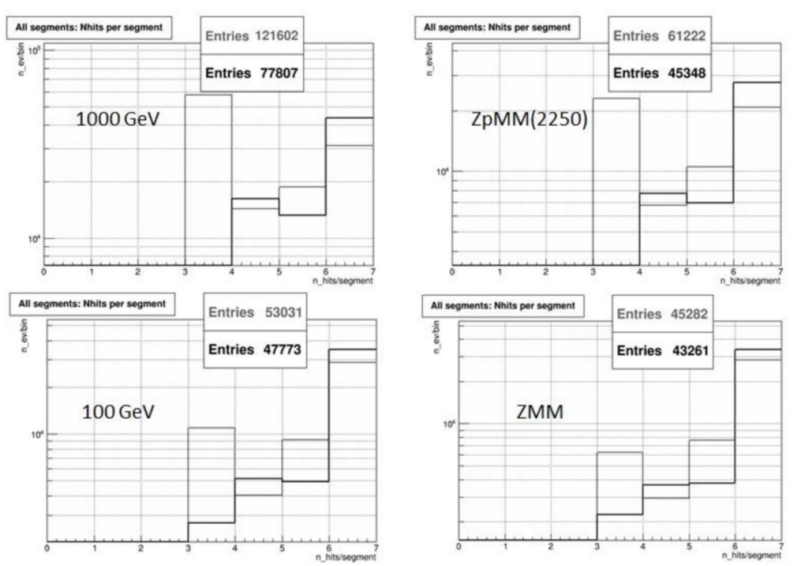

Figure 5: Number of rechits in all reconstructed segments for simulated data

Only one segment is chosen from each station in order to be matched with the muon and it is called a "muon segment". The number of hits per muon segment is presented in the Figure 6. The number of full (6-hit) muon segments increased for the new algorithm for all datasets while the amount of 
the smaller size muon segments decreased. This should result in a more precise reconstruction of the muon trajectory in the endcap muon system. The entry values form the statistics box show that the new algorithm reconstructs muon segments in some of the stations where the standard doesn't.
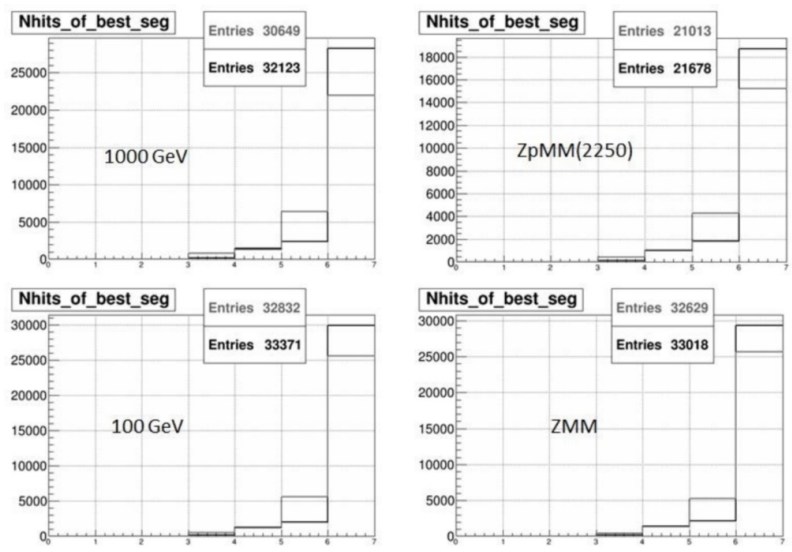

Figure 6: Number of rechits in the reconstructed muon segments for simulated data

We introduced the term of a "good" rechit in order to be able to evaluate the efficiency of the muon segment reconstruction. A "good" rechit is a rechit that is close enough to the simulated hit both for precise and rough coordinates. If there is more than one hit that satisfies this criterion, the closest to the simulated hit in terms of the Euclidian norm is taken for a "good" rechit.

The muon segment reconstruction efficiency was calculated only for the stations where at least 3 "good" rechits were found. This criterion was chosen in order to consider only the cases where it is possible to reconstruct at least 3-hit muon segments.

The change in efficiency with the increase of the values of the pseudorapidity $\eta$ is another interesting result, see Figure 7. Once the pseudorapidity increases, the rechit efficiency of the standard algorithm decreases especially for $\mathrm{TeV}$ muons. For the new algorithm it remains almost constant. It can be seen that the new algorithm is more than twice less inefficient than the standard one for all data samples. For example for the $Z^{\prime} \rightarrow \mu \mu$ sample the inefficiency of the standard algorithm equals $8 \%$ while that of the new algorithm is $3.7 \%$.

The distance between the reconstructed muon segment and the simulated muon track must be as small as possible in order to consider the results of the reconstruction reasonable and true. The differences between the simulated and reconstructed segment in terms of the azimuthal coordinate $\phi$ is presented in the Figure 8. The heavy tails of the distribution for this coordinate disappeared causing the decrease in the root mean square values (RMS). The most significant decrease was obtained for the $1000 \mathrm{GeV} / \mathrm{c}$ sample (3.6 times smaller RMS). There was also achieved a slight improvement for the polar coordinate $\theta$ but not as essential as in the case of $\phi$. It shows that the new algorithm reconstructs the muon segments closer to the muon trajectory as compared to that the standard algorithm.

\section{Conclusions}

The comparison of the results for different types of Monte-Carlo datasets shows that the new CSC segment building algorithm works well for all types of muon objects provided by the Large Hadron Collider beam collisions. The multiplicity of the reconstructed segments per station decreases mainly due to the suppression of the 3-hit segments by the new algorithm especially for $\mathrm{TeV}$ muons while 

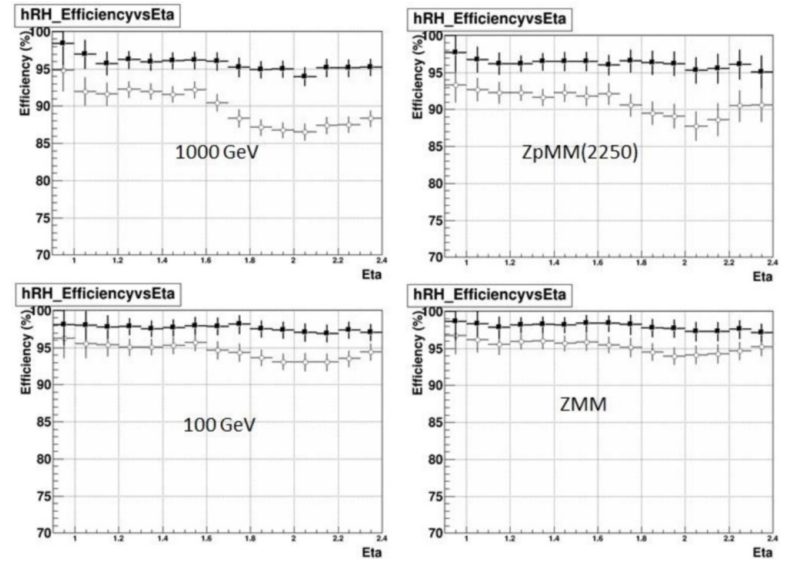

Figure 7: Muon segment reconstruction efficiency vs pseudorapidity $\eta$ for simulated data
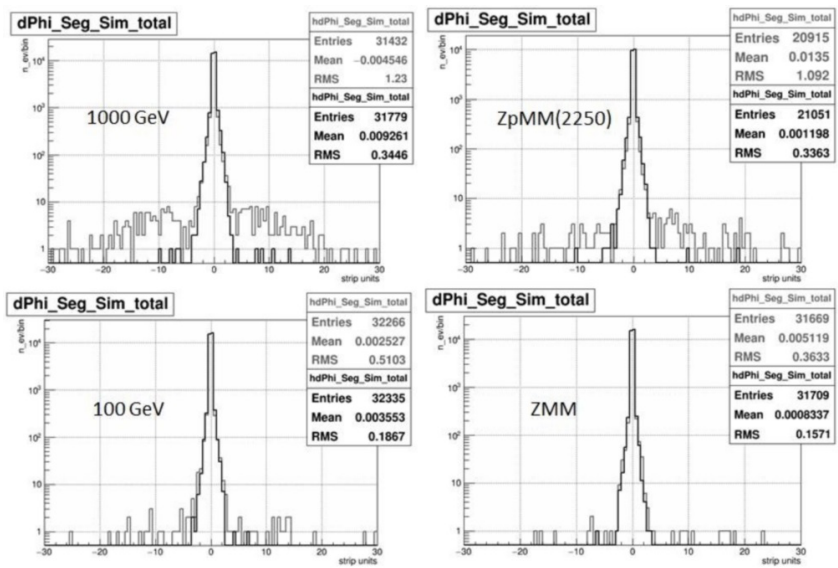

Figure 8: Difference between reconstructed and simulated azimuthal coordinate for simulated data

the ratio of the full (6-hit) segments increases for all datasets. The muon segment reconstruction inefficiency becomes more than twice smaller. The difference in the azimuthal coordinate between the reconstructed and the simulated muon segment gets much smaller that should result in a more precise and accurate reconstruction of the endcap muon transverse momentum.

The new algorithm appears to be stable and robust to hard muons and high luminosity which is expected in the Run2 of the LHC and beyond.

\section{References}

[1] CMS Collaboration, CMS Technical Proposal (CERN-LHCC-94-38, 1994)

[2] D. Harmon (ed.), Special issue on digital pattern recognition, Proc. IEEE 60, 1117 (1972)

[3] CMS Collaboration, CMS Physics TDR, Volume II (CERN-LHCC-2006-021, 2006)

[4] D. J. Hudson, Lectures on Elementary Statistics and Probability (CERN-63-29, 1963) 